\title{
Hydrogen peroxide induced loss of heterozygosity correlates with replicative lifespan and mitotic asymmetry in Saccharomyces cerevisiae
}

Emine Guven ${ }^{1,2}$, Lindsay A. Parnell ${ }^{1,3}$, Erin D Jackson ${ }^{1}$, Meighan C Parker ${ }^{1}$, Nilin Gupta ${ }^{1}$, Jenny Rodrigues 1, Hong Qin ${ }^{\text {Corresp. 1, } 4}$

${ }^{1}$ Biology, Spelman College, Atlanta, Georgia, United States

2 Current address: Computer Science and Engineering, University of Tennessee - Chattanooga, Chattanooga, Tennessee, United States

${ }^{3}$ Current address: Program of Molecular Genetics and Genomics, Division of Biology and Biomedical Sciences, Washington University in St. Louis, St. Louis, Missouri, United States

${ }^{4}$ Current address: Department of Computer Science and Engineering, Department of Biology, Geology, and Environmental Science, University of Tennessee - Chattanooga, Chattanooga, Tennessee, United States

Corresponding Author: Hong Qin

Email address: hqin@spelman.edu

Cellular aging in Saccharomyces cerevisiae can lead to genomic instability and impaired mitotic asymmetry. To investigate the role of oxidative stress in cellular aging, we examined the effect of exogenous hydrogen peroxide on genomic instability and mitotic asymmetry in a collection of yeast strains with diverse backgrounds. We treated yeast cells with hydrogen peroxide and monitored the changes of viability and the frequencies of loss of heterozygosity ( $\mathrm{LOH}$ ) in response to hydrogen peroxide doses. The mid-transition points of viability and LOH were quantified using sigmoid mathematical functions. We found that the increase of hydrogen peroxide dependent genomic instability often occurs before a drop in viability. We previously observed that elevation of genomic instability generally lags behind the drop in viability during chronological aging. Hence, onset of genomic instability induced by exogenous hydrogen peroxide treatment is opposite to that induced by endogenous oxidative stress during chronological aging, with regards to the midpoint of viability. This contrast argues that the effect of endogenous oxidative stress on genome integrity is well suppressed up to the dying-off phase during chronological aging. We found that the leadoff of exogenous hydrogen peroxide induced genomic instability to viability significantly correlated with replicative lifespan, indicating that yeast cells' ability to counter oxidative stress contributes to their replicative longevity. Surprisingly, this leadoff is positively correlated with an inverse measure of endogenous mitotic asymmetry, indicating a trade-off between mitotic asymmetry and cell's ability to fend off hydrogen peroxide induced oxidative stress. Overall, our results demonstrate strong associations of oxidative stress to genomic instability and mitotic asymmetry at the population level of budding yeast. 
1 Hydrogen peroxide induced loss of heterozygosity correlates with replicative lifespan and

2 mitotic asymmetry in Saccharomyces cerevisiae.

3 Emine Guven ${ }^{1,2,3}$, Lindsay A. Parnell ${ }^{1,2,4}$, Erin D. Jackson ${ }^{1}$, Meighan Parker ${ }^{1}$, Nilin Gupta ${ }^{1}$,

4 Jenny Rodrigues ${ }^{1}$, Hong Qin ${ }^{1,4,5}$

$5 \quad{ }^{1}$ Biology Department, Spelman College, Atlanta, GA 30314, United States

$6 \quad{ }^{2}$ Co-first authors.

$7{ }^{3}$ Current address: Department of Computer Science and Engineering, University of Tennessee at

8 Chattanooga, Chattanooga, TN, 37403, United States.

$9{ }^{4}$ Current address: Program of Molecular Genetics and Genomics, Division of Biology and

10 Biomedical Sciences, Washington University in St. Louis, St. Louis, Missouri 63110.

$11{ }^{5}$ Current address: Department of Biology, Geology, and Environmental Science, University of

12 Tennessee at Chattanooga, Chattanooga, TN, 37403, United States.

14 Corresponding Author:

15 Hong Qin

16 University of Tennessee at Chattanooga, Chattanooga, TN, 37403, United States.

17 Email: hong-qin@utc.edu

18 Short title: $\mathrm{H}_{2} \mathrm{O}_{2}$ induced genomic instability. 


\section{Abstract}

20

21

22

23

24

Cellular aging in Saccharomyces cerevisiae can lead to genomic instability and impaired mitotic asymmetry. To investigate the role of oxidative stress in cellular aging, we examined the effect of exogenous hydrogen peroxide on genomic instability and mitotic asymmetry in a collection of yeast strains with diverse backgrounds. We treated yeast cells with hydrogen peroxide and monitored the changes of viability and the frequencies of loss of heterozygosity (LOH) in response to hydrogen peroxide doses. The mid-transition points of viability and LOH were quantified using sigmoid mathematical functions. We found that the increase of hydrogen peroxide dependent genomic instability often occurs before a drop in viability. We previously observed that elevation of genomic instability generally lags behind the drop in viability during chronological aging. Hence, onset of genomic instability induced by exogenous hydrogen peroxide treatment is opposite to that induced by endogenous oxidative stress during chronological aging, with regards to the midpoint of viability. This contrast argues that the effect of endogenous oxidative stress on genome integrity is well suppressed up to the dying-off phase during chronological aging. We found that the leadoff of exogenous hydrogen peroxide induced genomic instability to viability significantly correlated with replicative lifespan, indicating that yeast cells' ability to counter oxidative stress contributes to their replicative longevity. Surprisingly, this leadoff is positively correlated with an inverse measure of endogenous mitotic asymmetry, indicating a trade-off between mitotic asymmetry and cell's ability to fend off hydrogen peroxide induced oxidative stress. Overall, our results demonstrate strong associations of oxidative stress to genomic instability and mitotic asymmetry at the population level of budding yeast.

\section{Key words:}


42 Aging, yeast, oxidative stress, lifespan, loss of heterozygosity, hydrogen peroxide

43

44

45

46

47

\section{Introduction}

The budding yeast Saccharomyces cerevisiae is a model for cellular aging (Kaeberlein 2010; Ludovico et al. 2012). Yeast aging can be studied by replicative aging and chronological aging based on dividing and non-dividing cells, respectively (Fabrizio \& Longo 2003; Longo et al. 2012). Replicative lifespan (RLS) refers to the number of times a cell undergoes the cell cycle (Defossez et al. 1998; Qin \& Lu 2006; Wei et al. 2008). Chronological lifespan (CLS) measures the amount of time it takes for cells to lose their proliferation potential in stationary phase (Fabrizio \& Longo 2003; Qin \& Lu 2006; Qin et al. 2008). Both replicative aging and chronological aging of yeast cells lead to increased genomic instability, as demonstrated by the age-dependent increase of loss of heterozygosity (LOH) (McMurray \& Gottschling 2003; McMurray \& Gottschling 2004; Qin et al. 2008). As a cellular organism, it is in the best interest for yeast mother cells to keep oxidatively damaged molecules to themselves, rather than pass them down to daughter cells, a phenomenon known as mitotic asymmetry (Henderson et al. 2014; Higuchi-Sanabria et al. 2014; Hill et al. 2016; Jazwinski 1990; Kennedy et al. 1994; Lai et al. 2002; McFaline-Figueroa et al. 2011; Thayer et al. 2014; Yang et al. 2015). Mitotic asymmetry can break down in both replicatively and chronologically aged cells, and lead to high levels of genomic instability in daughter cells (Henderson \& Gottschling 2008; McMurray \& Gottschling 2003; McMurray \& Gottschling 2004; Qin et al. 2008).

The free radical theory of aging argues that biological systems age because free radicals react with macromolecules and disturb key pathways that are vital to maintaining the overall functional and genomic integrity of cells (Harman 1956; Ristow \& Schmeisser 2011; Yu et al. 
2012). Reactive oxygen species (ROS), a form of free radicals, are natural by-products of the mitochondrial respiratory chain (Cheeseman \& Slater 1993). The endogenous levels of ROS, such as superoxide anions and hydrogen peroxide, are often low and play important roles in signaling transduction, defense, and other normal cell functions (Blagosklonny 2008; Rahman 2007; Weinberger et al. 2010). At low levels, hydrogen peroxide can promote yeast chronological longevity (Mesquita et al. 2010), a phenomenon known as hormesis (Belz \& Piepho 2012; Blagosklonny 2008; Blagosklonny 2009; Boxenbaum 1991; Ludovico \& Burhans 2014; Martins et al. 2014; Ristow \& Schmeisser 2011; Weinberger et al. 2007). At high levels, superoxide, hydrogen peroxide, and singlet oxygen can oxidize lipids, proteins, and nucleic acids (Moradas-Ferreira et al. 1996). The impaired cellular structures and functions induced by damaged molecules can result in altered metabolic homeostasis and abnormal cell growth, which has been argued to be a cause of aging (Harman 1956; Ristow \& Schmeisser 2011).

Substantial evidence support the role of ROS in yeast replicative aging and asymmetric age partition between mother and daughter cells (Higuchi-Sanabria et al. 2014). Replicatively older cells showed higher levels of superoxide anion and hydrogen peroxide than younger cells, as measured by dihydroethidium and dihydrorhodamine, respectively (Lam et al. 2011; Scheckhuber et al. 2007). Mitochondria in mother cells were found to gradually lose their inner membrane potential and lead to an iron-sulfur cluster defect and nuclear genome instability (Veatch et al. 2009). This mitochondria dysfunction can lead to increased levels of ROS (Breitenbach et al. 2014). Mother cells tend to retain mitochondria with higher superoxide levels than those in daughter cells (McFaline-Figueroa et al. 2011). The asymmetric partition of mitochondria between mother and daughter cells can be enhanced by increased retrograde actin cable flow (Higuchi et al. 2013). Consistent with the role of ROS, decreased release of 
88 mitochondrial reactive oxygen can extend replicative lifespan (Barros et al. 2004), and 89 supplementation of spermine/spermidine, free-radical scavengers, can increase yeast replicative 90 lifespan (Eisenberg et al. 2009).

91 The roles of superoxide anion and hydrogen peroxide in yeast chronological aging have 92 also been investigated. Calorie restriction is found to extend chorological lifespan through 93 moderate increase of hydrogen peroxide that leads to activation of superoxide dismutase and 94 inhibition of superoxide anions (Mesquita et al. 2010; Weinberger et al. 2010). Heavy water can suppress endogenous ROS and extend yeast chronological lifespan (Li \& Synder 2016).

We previously found that yeast natural isolates tend to hold off the age-dependent increase of LOH during chronological aging, as shown by the late-onset of LOH increase when compared to the drop of viability (Qin et al. 2008), and this ability is significantly associated with yeast replicative lifespan and a measure of mitotic asymmetry (Qin et al. 2008). The chronological lifespan dependent change of LOH was independently observed by another research group (Maxwell et al. 2011). Age-dependent genomic instability has been attributed to oxidative damage to chromosomal DNA (Burhans \& Weinberger 2007; Burhans \& Weinberger 2012; McMurray \& Gottschling 2004; Weinberger et al. 2007).

In the present study, we seek to test whether there is a detectable statistical link between yeast lifespan variations and oxidative stress induced genomic instability at the population level.

\section{Materials and Methods}

\section{Yeast strains and $\mathrm{H}_{2} \mathrm{O}_{2}$ treatment}

Strains with $M E T 15^{+/-}$have been described previously (Qin et al. 2008). Yeast cells were grown overnight at $30^{\circ} \mathrm{C}$ in YPD. On the next day, yeast cultures were diluted to $\mathrm{OD}_{600}$ of 0.6 in 
110 fresh YPD and grown for an additional two hours to ensure that the optical density reached

111 between 0.8 and 0.9 , the mid-log phase. Cells were then harvested, washed with water to remove

112 YPD, and sonicated for 4 minutes using a Fisher Scientific FS20D water-bath sonicator to

113 disperse cell clumps.

114 Treatment of yeast cells with $\mathrm{H}_{2} \mathrm{O}_{2}$ was modified from other studies (Diezmann \&

115 Dietrich 2009; Yu et al. 2012). Reactions were carried out by mixing $4 \mu 1$ of yeast cells, $16 \mu l$ of 116 water, and $20 \mu \mathrm{l}$ of the 2 -fold $\mathrm{H}_{2} \mathrm{O}_{2}$ working stocks. Tubes of cells in $\mathrm{H}_{2} \mathrm{O}_{2}$ treatment were 117 incubated on a nutator for 3 hours at $30^{\circ} \mathrm{C}$. For most strains, the final concentrations of $\mathrm{H}_{2} \mathrm{O}_{2}$ for 118 cell treatment were typically in a group of ten: $0 \%, 0.005 \%, 0.01 \%, 0.025 \%, 0.05 \%, 0.075 \%$, $1190.1 \%, 0.15 \%, 0.2 \%$, and $0.3 \%$. For strains that are more sensitive or more tolerant to $\mathrm{H}_{2} \mathrm{O}_{2}$, this 120 concentration series was adjusted lower or higher, respectively. At the end of incubation, all 121 reaction tubes were filled with $960 \mu \mathrm{l}$ of water (a 50 fold dilution) and chilled on ice for 5 122 minutes. Cells were sonicated in a FS20D water-bath sonicator for 2 minutes. We spread $250 \mu 1$ 123 of each reaction mix onto $150 \mathrm{~mm}$ plates with the lead-containing media MLA (Qin et al. 2008).

124 Plates were spread in triplicates for each $\mathrm{H}_{2} \mathrm{O}_{2}$ concentration. Plates were incubated at $30^{\circ} \mathrm{C}$ for 2 125 days. Some plates were also left at room temperature for additional days for colony colors to 126 develop a better contrast for LOH scoring. Colonies were visually assessed for color-sectoring 127 patterns and tallied using a Bantex Colony Counter. We tallied the number of white, black, half128 black, quarter-black, three-quarter black colonies and others. We ignored color-sector patterns 129 that were less than one-eighth. For each experiment, viability at conditions with only water was 130 deemed as $100 \%$. Viability at a specific concentration of $\mathrm{H}_{2} \mathrm{O}_{2}$ was estimated from the average 131 number of colony forming units (CFUs) at this concentration divided by the average number of 
132 CFUs at conditions with only water. Strains were assayed 2 - 5 times, for a total of 35 successful 133 experiments performed with the 12 yeast strains under study.

\section{Data and analysis codes}

Fitting and analysis were conducted in the R statistical environment. Sample codes and data can be found at https://github.com/hongqin/H2O2LOH PeerJ2016. An R markdown script, "summary_analysis_LOHH2O2.Rmd", is provided in the GitHub repository to reproduce key analyses and figures. A summary of the results in EXCEL format is provided in Supplementary Table $\mathrm{S} 1$. The ratios of $\mathrm{C}_{\mathrm{b}} / \mathrm{C}_{\mathrm{v}}$ were directly estimated from each experiment and then averaged for each strain. Data for replicative and chronological lifespan, and mitotic asymmetry measures were obtained from our previous studies (Qin \& Lu 2006; Qin et al. 2008), and are also available in the same GitHub repository. ARLS indicates the average replicative lifespan for each strain that was previously measured using micro-dissections (Qin \& Lu 2006). CLS in the dataset indicates average chronological lifespan for each strain that was previously measured using colony-forming units (Qin \& Lu 2006).

\section{Results}

\section{Overview of the experiment}

To better understand the interconnection between oxidative stress, genomic instability, mitotic asymmetry, and chronological lifespan in $\mathrm{S}$. cerevisiae, we used exogenous $\mathrm{H}_{2} \mathrm{O}_{2}$ to

152 induce an oxidative stress response in yeast cells and quantified their $\mathrm{H}_{2} \mathrm{O}_{2}$ dose-response curves of $\mathrm{LOH}$ and viability. $\mathrm{LOH}$ is an effective method for gauging genome integrity in yeast cells

154 (McMurray \& Gottschling 2003; McMurray \& Gottschling 2004). We previously introduced 
155 heterozygosity on the MET15 locus (MET15+/) by knocking out one copy of the wild-type allele

156 using a kanamycin-resistance marker (Qin et al. 2008). LOH can be monitored when MET15

157 strains are converted into homozygous $\mathrm{MET15}^{-/}$strains that can generate black colors on lead-

158 containing medium (Figure 1). In other words, we can observe half of the LOH events at the $159 \mathrm{MET15}^{+/-}$locus.

160

\section{Quantitative measurement of $\mathrm{H}_{2} \mathrm{O}_{2}$-dose dependent changes of viability and $\mathrm{LOH}$}

S1). Half-black colonies indicated LOH occurred after cells divided on MLA plates (Figure 1).

As shown in Figure 2, we quantified viability $s$ as a function of hydrogen peroxide concentration, $\left[\mathrm{H}_{2} \mathrm{O}_{2}\right]$, using a sigmoid mathematical function, similar to our previous study (Qin et al. 2008) :

$$
s\left(\left[\mathrm{H}_{2} \mathrm{O}_{2}\right]\right)=\frac{1}{1+\left(\frac{\left[\mathrm{H}_{2} \mathrm{O}_{2}\right]}{C_{v}}\right)^{w_{v}}},
$$

where $\mathrm{C}_{\mathrm{v}}$ is the $\mathrm{H}_{2} \mathrm{O}_{2}$ concentration at the midpoint of viability, and $\mathrm{w}_{\mathrm{v}}$ is a weight parameter.

We quantified the percentage of black colonies, $\mathrm{b}\left(\left[\mathrm{H}_{2} \mathrm{O}_{2}\right]\right)$, as a function of $\left[\mathrm{H}_{2} \mathrm{O}_{2}\right]$ :

$$
\mathrm{b}\left(\left[\mathrm{H}_{2} \mathrm{O}_{2}\right]\right)=\mathrm{b}_{\max }-\frac{\mathrm{b}_{\max }-\mathrm{b}_{\min }}{1+\left(\frac{\left[\mathrm{H}_{2} \mathrm{O}_{2}\right]}{C_{b}}\right)^{w_{b}}},
$$

where $\mathrm{C}_{\mathrm{b}}$ is the $\mathrm{H}_{2} \mathrm{O}_{2}$ concentration at the midpoint of black colony change, $b_{\max }$ and $b_{\min }$ is the

173 maximum and minimum of $\mathrm{b}\left(\left[\mathrm{H}_{2} \mathrm{O}_{2}\right]\right)$, and $\mathrm{w}_{\mathrm{b}}$ is a weight parameter. Likewise, the change of

174 half-black colonies was quantified: 


$$
b_{0.5}\left(\left[\mathrm{H}_{2} \mathrm{O}_{2}\right]\right)=b_{0.5 \max }-\frac{\mathrm{b}_{0.5 \max }-\mathrm{b}_{0.5 \min }}{1+\left(\frac{\left[\mathrm{H}_{2} \mathrm{O}_{2}\right]}{C_{b 0.5}}\right)^{w_{b 0.5}}}
$$

176 where $\mathrm{C}_{\mathrm{b} 0.5}$ is the $\mathrm{H}_{2} \mathrm{O}_{2}$ concentration at the midpoint of half-black colony change.

177 We detected a strong association between $\mathrm{C}_{\mathrm{v}}$ and $\mathrm{C}_{\mathrm{b}}$ with $\mathrm{R}^{2}=0.77$ and p-value $=$ 1780.00019 using the averaged values of each strain (Supplemental Figure S1), indicating that 179 hydrogen peroxide induced LOH and loss of viability co-vary, demonstrating the consistency of 180 our approach.

\section{Genomic tolerance to oxidative stress correlates with replicative lifespan}

$\mathrm{C}_{\mathrm{b}}$ is lower than $\mathrm{C}_{\mathrm{v}}$ in 7 out of the 12 strains under study (Figure 2, Supplemental Table $\mathrm{S} 1$, Supplemental Figure $\mathrm{S} 2$ ). The median and mean values of $\mathrm{C}_{\mathrm{b}} / \mathrm{C}_{\mathrm{v}}$ are 0.60 and 0.92 , respectively, indicating that lower doses of $\mathrm{H}_{2} \mathrm{O}_{2}$ are needed to trigger genomic instability than to trigger the drop of viability. In other words, genome integrity is more sensitive to oxidative stress than cellular viability during exogenous $\mathrm{H}_{2} \mathrm{O}_{2}$ treatment. Strains with higher $\mathrm{C}_{\mathrm{b}} / \mathrm{C}_{\mathrm{v}}$ values, such as YPS128 in Figure 3A, indicates their genome integrity is more tolerant of hydrogen peroxide. Strains with lower $C_{b} / C_{v}$ values, such as M32 in Figure 3A, indicate their genome integrity is more sensitive to hydrogen peroxide. Linear regression analysis reveals that average replicative lifespan, ARLS, is positively correlated with $\mathrm{C}_{\mathrm{b}} / \mathrm{C}_{\mathrm{v}}$ with $\mathrm{p}$-value $=0.039$ and $\mathrm{R}^{2}=0.36$, suggesting that genomic tolerance to oxidative stress is associated with replicative longevity. 
193 Trade-off between genomic tolerance to oxidative stress and mitotic asymmetry in mother 194 cells

We previously measured an inverse proxy for the mitotic asymmetry, $\mathrm{L}_{0}$, of these yeast 196 strains during normal growth conditions (Qin et al. 2008). This inverse proxy for mitotic 197 asymmetry, $\mathrm{L}_{0}$, is the ratio of daughter-cell LOH versus mother-cell LOH in YPD. Small $\mathrm{L}_{0}$ 198 values suggest mother cells can more effectively keep genotoxic stress factors to themselves and 199 thereby provide their daughter cells with healthier lives (Qin et al. 2008). Regression analysis 200 revealed that $\mathrm{C}_{\mathrm{b}} / \mathrm{C}_{\mathrm{v}}$ significantly correlates with $\mathrm{L}_{0}$, with $\mathrm{p}$-value $=0.0079$ and $\mathrm{R}^{2}=0.56$ (Figure 201 3B). Based on the positive correlation between $C_{b} / C_{v}$ and $L_{0}$, yeast cells with better mitotic 202 asymmetry, such as M1-2 and M13, are more sensitive to genotoxic stress because relatively 203 lower $\mathrm{H}_{2} \mathrm{O}_{2}$ can trigger $\mathrm{LOH}$ in their genomes. Hence, better abilities of mother cells to prevent 204 genotoxic stress factors from leaking to daughter cells also render mothers' own genomes more 205 sensitive to elevated oxidative stress.

\section{Concerted increase of genomic instability}

We observed a large number of multiple $\mathrm{LOH}$ events where $\mathrm{H}_{2} \mathrm{O}_{2}$ induced $\mathrm{LOH}$ occurred in both mother and daughter cells. We focused on the three-quarter black colonies to illustrate the nature of concerted multiple $\mathrm{LOH}$ events. Based on parsimonious reasoning, a three-quarter black LOH can be considered as a joint outcome of a half-black and a quarter-black LOH. The null hypothesis here is that half-black LOH and quarter-black LOH occur independently, which means that the probability of three-quarter black colonies is the product of the probabilities of

214 half- and quarter-blacks, i.e. random expectation. The alternative hypothesis is that events of 215 half-black $\mathrm{LOH}$ and quarter-black $\mathrm{LOH}$ are associated with and lead to an increased occurrence 
216 of three-quarter black LOH. We performed one-tailed Fisher's exact tests on experimental

217 observations with sufficient events of half-, quarter-, and three-quarter black LOH events, and

218 applied Bonferroni correction to adjust for multiple tests. Of the 35 experiments, 21 experiments

219 show that three-quarter $\mathrm{LOH}$ events occurred at significantly higher frequencies in all $\mathrm{H}_{2} \mathrm{O}_{2}$

220 conditions with sufficient number of LOH events (Supplemental Table S2). In nine experiments,

221 three-quarter $\mathrm{LOH}$ events occurred at significantly higher frequencies in all but one $\mathrm{H}_{2} \mathrm{O}_{2}$

222 condition. Overall, 163 out of 185 measurements show significantly higher three-quarter LOH

223 events than the null hypothesis (Supplemental Table S2). Hence, the null hypothesis can

224 generally be rejected. We concluded that the high occurrence of three-quarter black colonies is

225 the result of multiple concerted LOH events.

Contrasting LOH onsets between hydrogen peroxide treatment and chronological aging suggest cells' ability to suppress endogenous oxidative stress during aging

In our previous study on $\mathrm{LOH}$ during chronological aging, we found that the onset of increase of $\mathrm{LOH}$, and $\mathrm{T}_{\mathrm{c}}$ as the midpoint for the drop of viability during chronological aging (Figure 4) (Qin et al. 2008). $\mathrm{T}_{\mathrm{g}}$ values are often larger than $\mathrm{T}_{\mathrm{c}}$ values in the collection of yeast strains that we studied (Figure 4) (Qin et al. 2008). of viability, as $C_{b}$ is often smaller than $C_{v}$ (Figure 4). For most natural isolates, this $C_{b} / C_{v}$ ratio is lower than 1.0 (Figure 4). If we assume that a similar level of oxidative stress corresponds to the $50 \%$ viability in each strain, this contrast suggests that during chronological aging, the genotoxic effect of endogenous oxidative stress is shielded even when viability has dropped during 
239 chronological aging. Hence, the contrast between LOH onsets is a proxy for the extent to how

240 much cells can suppress the genotoxic effect of endogenous oxidative stress on genome integrity.

241 Based on this reasoning, the ratio of $\frac{T_{g} / T_{c}}{C_{b} / C_{v}}$ indicates the ability of yeast cells to suppress the level

242 of endogenous oxidative stress during the normal chronological aging process. Remarkably, we

243 found a trade-off between mitotic asymmetry and ability of cells to suppress endogenous

244 oxidative stress during chronological aging, as shown by a negative linear correlation between

245 mitotic asymmetry $\mathrm{L}_{0}$ and $\frac{T_{g} / T_{c}}{C_{b} / C_{v}}$ (Figure 4D). This finding corroborates the tradeoff observed in

246 Figure 3B.

248 Summary and Discussion

Our results show that exogenous $\mathrm{H}_{2} \mathrm{O}_{2}$ tends to induce early-onset of $\mathrm{LOH}$ increase, in contrast to LOH events caused by endogenous oxidative stress during chronological aging. We found that the tolerance of yeast strains to exogenous $\mathrm{H}_{2} \mathrm{O}_{2}$ correlated positively with their average replicative lifespan and trade-off with mitotic asymmetry. We argue that the contrasting onsets of LOH caused by exogenous and endogenous oxidative stress indicate the ability of cells to suppress genotoxic stress during chronological aging, and this contrast correlates well with mitotic asymmetry. We concluded that a yeast strain's ability to counter genotoxic stress is a good predictor of its replicative lifespan and its mitotic asymmetry, although the connection between replicative lifespan and mitotic asymmetry is more complicated (Figure 5A). We presented a plausible explanation to account for the trade-off between genomic tolerance to 
260 stronger ability to prevent oxidative damages from leaking to the daughter cells, mother cells put

261 their own nuclear genomes at a higher risk for oxidative damage (Figure 5B). When mother cells

262 tend to leak more oxidative damages to daughter cells, mother cells have a better chance to

263 protect the integrity of their genomes (Figure 5C).

264 Strong evidence supports the role of reactive oxygen species in yeast replicative aging

265 and asymmetric age partition between mother and daughter cells. Replicatively older cells

266 showed higher levels of superoxide anion and $\mathrm{H}_{2} \mathrm{O}_{2}$ than younger cells, as measured by

267 dihydroethidium and dihydrorhodamine, respectively (Lam et al. 2011; Scheckhuber et al. 2007).

268 Mitochondria in mother cells were found to gradually lose their inner membrane potential, lead

269 to an iron-sulfur cluster defect and nuclear genome instability (Veatch et al. 2009). This

270 mitochondria dysfunction can lead to increased levels of ROS (Breitenbach et al. 2014). Mother

271 cells tend to retain mitochondria with higher superoxide levels than those in daughter cells

272 (McFaline-Figueroa et al. 2011). Consistent with the role of ROS, supplementation of

273 spermine/spermidine, free-radical scavengers, can increase yeast replicative lifespan (Eisenberg

274 et al. 2009). We speculate that exogenous hydrogen peroxide most likely disrupts the

275 homeostasis of endogenous reactive oxygen species and might in turn lead to high levels of

276 superoxide anions. Clearly, further studies are needed to verify these conjectures.

277 We would like to emphasize that our main conclusions in Figure 5 should be viewed as a

278 statistical generalization at the population level and are an indirect observation of the role of

279 ROS in yeast aging. Yeast lifespan is a known pleiotropic trait with a large range of phenotypic

280 plasticity. In 'wildtype' yeast strains, the average replicative lifespan can vary by more than 2-

281 fold, and the average chronological lifespan can change by nearly 5-fold (Qin et al. 2008). The

282 detailed pictures for the role of reactive oxygen species in yeast aging are fairly complicated at 
283 molecular levels (Barros et al. 2004; Lin et al. 2002; Mesquita et al. 2010; Reverter-Branchat et

284 al. 2004; Weinberger et al. 2010), and part of the complexity can be attributed to ecological

285 niches and genetic histories of yeast strains. Recently, several quantitative models related to

286 yeast aging have been developed, though much work is needed to improve these models

287 (Gillespie et al. 2004; Kowald \& Kirkwood 1994; Rashidi et al. 2012; Sozou \& Kirkwood 2001;

288 Tan et al. 2009; Tang \& Liu 2010). By looking at the statistical trends at the population level, we

289 show a tradeoff between oxidative stress response and mitotic asymmetry. This tradeoff would

290 be difficult to discern at the molecular level in individual strains. We hope our findings (Figure

291 5) will be useful for developing improved mathematical models on yeast aging.

The MET15 locus is located adjacent to the rDNA locus on chromosome 12, as we 293 discussed previously (Qin et al. 2008). Accumulation of extrachromosomal rDNA was shown to cause replicative aging (Sinclair \& Guarente 1997). Recently, it was argued that stability of the rDNA locus is the main determinant of yeast lifespan (Ganley et al. 2009; Kobayashi 2014), and rDNA locus stability can be influenced by more than $10 \%$ of yeast genes (Saka et al. 2016). Increased genomic instability in aging mother cells correlates with increased insertions of retrotransposons at the rDNA locus (Patterson et al. 2015). Further studies are needed to investigate the link between oxidative response and rDNA locus, perhaps through nextgeneration sequencing of multiple yeast strains.

Based on the observed distribution of $C_{b} / C_{v}$ in the 12 strains studied (Figure 4B), there 302 may be two groups of yeast strains: One group with $\mathrm{C}_{\mathrm{b}} / \mathrm{C}_{\mathrm{v}}$ greater than 1 , and one group with $\mathrm{C}_{\mathrm{b}} / \mathrm{C}_{\mathrm{v}}$ less than 1 . We argue that more yeast strains need to be surveyed in order to address this 304 question. 
307 yeast strains with sequenced genomes (Kaya et al. 2015), Saccharomyces cerevisiae has become 308 a promising model organism to understand cellular aging through the integration of molecular 309 biology, population biology, and systems biology. 
312

313

314

315

316

317

318

319

320

322

323

324

Reference:

Barros MH, Bandy B, Tahara EB, and Kowaltowski AJ. 2004. Higher respiratory activity decreases mitochondrial reactive oxygen release and increases life span in Saccharomyces cerevisiae. J Biol Chem 279:49883-49888.

Belz RG, and Piepho HP. 2012. Modeling effective dosages in hormetic dose-response studies. PLoS One 7:e33432.

Blagosklonny MV. 2008. Aging: ROS or TOR. Cell Cycle 7:3344-3354.

Blagosklonny MV. 2009. TOR-driven aging: speeding car without brakes. Cell Cycle 8:4055-4059.

Boxenbaum H. 1991. Gompertz mortality analysis: aging, longevity hormesis and toxicity. Arch Gerontol Geriatr 13:125-137.

Breitenbach M, Rinnerthaler M, Hartl J, Stincone A, Vowinckel J, Breitenbach-Koller H, and Ralser M. 2014. Mitochondria in ageing: there is metabolism beyond the ROS. FEMS Yeast Res 14:198-212.

Burhans WC, and Weinberger M. 2007. DNA replication stress, genome instability and aging. Nucleic Acids Res 35:7545-7556.

Burhans WC, and Weinberger M. 2012. DNA damage and DNA replication stress in yeast models of aging. In: Breitenbach M, ed. Aging Research in Yeast (Subcellular Biochemistry): Springer, 187206.

Cheeseman KH, and Slater TF. 1993. An introduction to free radical biochemistry. Br Med Bull 49:481493.

Defossez PA, Park PU, and Guarente L. 1998. Vicious circles: a mechanism for yeast aging. Curr Opin Microbiol 1:707-711.

Diezmann S, and Dietrich FS. 2009. Saccharomyces cerevisiae: population divergence and resistance to oxidative stress in clinical, domesticated and wild isolates. PLoS One 4:e5317.

Eisenberg T, Knauer H, Schauer A, Buttner S, Ruckenstuhl C, Carmona-Gutierrez D, Ring J, Schroeder S, Magnes C, Antonacci L, Fussi H, Deszcz L, Hartl R, Schraml E, Criollo A, Megalou E, Weiskopf D, Laun P, Heeren G, Breitenbach M, Grubeck-Loebenstein B, Herker E, Fahrenkrog B, Frohlich KU, Sinner F, Tavernarakis N, Minois N, Kroemer G, and Madeo F. 2009. Induction of autophagy by spermidine promotes longevity. Nat Cell Biol 11:1305-1314.

Fabrizio P, and Longo VD. 2003. The chronological life span of Saccharomyces cerevisiae. Aging Cell 2:7381.

Ganley AR, Ide S, Saka K, and Kobayashi T. 2009. The effect of replication initiation on gene amplification in the rDNA and its relationship to aging. Mol Cell 35:683-693. 
344

Gillespie CS, Proctor CJ, Boys RJ, Shanley DP, Wilkinson DJ, and Kirkwood TB. 2004. A mathematical model of ageing in yeast. J Theor Biol 229:189-196.

Harman D. 1956. Aging: a theory based on free radical and radiation chemistry. J Gerontol 11:298-300.

Henderson KA, and Gottschling DE. 2008. A mother's sacrifice: what is she keeping for herself? Curr Opin Cell Biol 20:723-728.

Henderson KA, Hughes AL, and Gottschling DE. 2014. Mother-daughter asymmetry of pH underlies aging and rejuvenation in yeast. Elife 3:e03504.

Higuchi R, Vevea JD, Swayne TC, Chojnowski R, Hill V, Boldogh IR, and Pon LA. 2013. Actin dynamics affect mitochondrial quality control and aging in budding yeast. Curr Biol 23:2417-2422.

Higuchi-Sanabria R, Pernice WM, Vevea JD, Alessi Wolken DM, Boldogh IR, and Pon LA. 2014. Role of asymmetric cell division in lifespan control in Saccharomyces cerevisiae. FEMS Yeast Res 14:1133-1146.

Hill SM, Hao X, Gronvall J, Spikings-Nordby S, Widlund PO, Amen T, Jorhov A, Josefson R, Kaganovich D, Liu B, and Nystrom T. 2016. Asymmetric Inheritance of Aggregated Proteins and Age Reset in Yeast Are Regulated by Vac17-Dependent Vacuolar Functions. Cell Rep 16:826-838.

Jazwinski SM. 1990. An experimental system for the molecular analysis of the aging process: the budding yeast Saccharomyces cerevisiae. J Gerontol 45:B68-74.

Kaeberlein M. 2010. Lessons on longevity from budding yeast. Nature 464:513-519.

Kaya A, Ma S, Wasko B, Lee M, Kaeberlein M, and Gladyshev VN. 2015. Defining Molecular Basis for Longevity Traits in Natural Yeast Isolates. NPJ Aging Mech Dis 1.

Kennedy BK, Austriaco NR, Jr., and Guarente L. 1994. Daughter cells of Saccharomyces cerevisiae from old mothers display a reduced life span. J Cell Biol 127:1985-1993.

Kobayashi T. 2014. Ribosomal RNA gene repeats, their stability and cellular senescence. Proc Jpn Acad Ser B Phys Biol Sci 90:119-129.

Kowald A, and Kirkwood TB. 1994. Towards a network theory of ageing: a model combining the free radical theory and the protein error theory. J Theor Biol 168:75-94.

Lai CY, Jaruga E, Borghouts C, and Jazwinski SM. 2002. A mutation in the ATP2 gene abrogates the age asymmetry between mother and daughter cells of the yeast Saccharomyces cerevisiae. Genetics 162:73-87.

Lam YT, Aung-Htut MT, Lim YL, Yang H, and Dawes IW. 2011. Changes in reactive oxygen species begin early during replicative aging of Saccharomyces cerevisiae cells. Free Radic Biol Med 50:963-970. 
Li X, and Synder MP. 2016. Yeast longevity promoted by reversing aging-associated decline in heavy isotope content. NPJ Aging Mech Dis 2.

Lin SJ, Kaeberlein M, Andalis AA, Sturtz LA, Defossez PA, Culotta VC, Fink GR, and Guarente L. 2002. Calorie restriction extends Saccharomyces cerevisiae lifespan by increasing respiration. Nature 418:344-348.

Longo VD, Shadel GS, Kaeberlein M, and Kennedy B. 2012. Replicative and chronological aging in Saccharomyces cerevisiae. Cell Metab 16:18-31.

Ludovico P, and Burhans WC. 2014. Reactive oxygen species, ageing and the hormesis police. FEMS Yeast Res 14:33-39.

Ludovico P, Osiewacz HD, Costa V, and Burhans WC. 2012. Cellular models of aging. Oxid Med Cell Longev 2012:616128.

Martins D, Titorenko VI, and English AM. 2014. Cells with impaired mitochondrial H2O2 sensing generate less * $\mathrm{OH}$ radicals and live longer. Antioxid Redox Signal 21:1490-1503.

Maxwell PH, Burhans WC, and Curcio MJ. 2011. Retrotransposition is associated with genome instability during chronological aging. Proc Natl Acad Sci U S A 108:20376-20381.

McFaline-Figueroa JR, Vevea J, Swayne TC, Zhou C, Liu C, Leung G, Boldogh IR, and Pon LA. 2011. Mitochondrial quality control during inheritance is associated with lifespan and motherdaughter age asymmetry in budding yeast. Aging Cell 10:885-895.

McMurray MA, and Gottschling DE. 2003. An age-induced switch to a hyper-recombinational state. Science 301:1908-1911.

McMurray MA, and Gottschling DE. 2004. Aging and genetic instability in yeast. Curr Opin Microbiol 7:673-679.

Mesquita A, Weinberger M, Silva A, Sampaio-Marques B, Almeida B, Leao C, Costa V, Rodrigues F, Burhans WC, and Ludovico P. 2010. Caloric restriction or catalase inactivation extends yeast chronological lifespan by inducing $\mathrm{H} 2 \mathrm{O} 2$ and superoxide dismutase activity. Proc Natl Acad Sci U S A 107:15123-15128.

Moradas-Ferreira P, Costa V, Piper P, and Mager W. 1996. The molecular defences against reactive oxygen species in yeast. Mol Microbiol 19:651-658.

Patterson MN, Scannapieco AE, Au PH, Dorsey S, Royer CA, and Maxwell PH. 2015. Preferential retrotransposition in aging yeast mother cells is correlated with increased genome instability. DNA Repair (Amst) 34:18-27.

Qin H, and Lu M. 2006. Natural variation in replicative and chronological life spans of Saccharomyces cerevisiae. Exp Gerontol 41:448-456. 
408

409

410

411

412

413

414

415

416

417

418

419

420

421

422

423

424

425

426

427

428

429

430

431

432

433

434

435

436

437

438

439

440

441

Qin H, Lu M, and Goldfarb DS. 2008. Genomic instability is associated with natural life span variation in Saccharomyces cerevisiae. PLoS One 3:e2670.

Rahman K. 2007. Studies on free radicals, antioxidants, and co-factors. Clin Interv Aging 2:219-236.

Rashidi A, Kirkwood TBL, and Shanley DP. 2012. Evolution of asymmetric damage segregation: A modeling approach. In: Breitenbach M, ed. Aging Research in Yeast (Subcellular Biochemistry): Springer, 315-330.

Reverter-Branchat G, Cabiscol E, Tamarit J, and Ros J. 2004. Oxidative damage to specific proteins in replicative and chronological-aged Saccharomyces cerevisiae: common targets and prevention by calorie restriction. J Biol Chem 279:31983-31989.

Ristow M, and Schmeisser S. 2011. Extending life span by increasing oxidative stress. Free Radic Biol Med 51:327-336.

Saka K, Takahashi A, Sasaki M, and Kobayashi T. 2016. More than $10 \%$ of yeast genes are related to genome stability and influence cellular senescence via rDNA maintenance. Nucleic Acids Res 44:4211-4221.

Scheckhuber CQ, Erjavec N, Tinazli A, Hamann A, Nystrom T, and Osiewacz HD. 2007. Reducing mitochondrial fission results in increased life span and fitness of two fungal ageing models. Nat Cell Biol 9:99-105.

Sinclair DA, and Guarente L. 1997. Extrachromosomal rDNA circles--a cause of aging in yeast. Cell 91:1033-1042.

Sozou PD, and Kirkwood TB. 2001. A stochastic model of cell replicative senescence based on telomere shortening, oxidative stress, and somatic mutations in nuclear and mitochondrial DNA. $J$ Theor Biol 213:573-586.

Tan Y, Bush JM, Liu W, and Tang F. 2009. Identification of longevity genes with systems biology approaches. Adv Appl Bioinform Chem 2:49-56.

Tang F, and Liu W. 2010. An age-dependent feedback control model of calcium dynamics in yeast cells. J Math Biol 60:849-879.

Thayer NH, Leverich CK, Fitzgibbon MP, Nelson ZW, Henderson KA, Gafken PR, Hsu JJ, and Gottschling DE. 2014. Identification of long-lived proteins retained in cells undergoing repeated asymmetric divisions. Proc Natl Acad Sci U S A 111:14019-14026.

Veatch JR, McMurray MA, Nelson ZW, and Gottschling DE. 2009. Mitochondrial dysfunction leads to nuclear genome instability via an iron-sulfur cluster defect. Cell 137:1247-1258.

Wei M, Fabrizio P, Hu J, Ge H, Cheng C, Li L, and Longo VD. 2008. Life span extension by calorie restriction depends on Rim15 and transcription factors downstream of Ras/PKA, Tor, and Sch9. PLoS Genet 4:e13. 
442 Weinberger M, Feng L, Paul A, Smith DL, Jr., Hontz RD, Smith JS, Vujcic M, Singh KK, Huberman JA, and

443

444

445

446

447

448

449

450

451

452

453

454

455

456

457
Burhans WC. 2007. DNA replication stress is a determinant of chronological lifespan in budding yeast. PLoS One 2:e748.

Weinberger M, Mesquita A, Caroll T, Marks L, Yang H, Zhang Z, Ludovico P, and Burhans WC. 2010. Growth signaling promotes chronological aging in budding yeast by inducing superoxide anions that inhibit quiescence. Aging (Albany NY) 2:709-726.

Yang J, McCormick MA, Zheng J, Xie Z, Tsuchiya M, Tsuchiyama S, El-Samad H, Ouyang Q, Kaeberlein M, Kennedy BK, and Li H. 2015. Systematic analysis of asymmetric partitioning of yeast proteome between mother and daughter cells reveals "aging factors" and mechanism of lifespan asymmetry. Proc Natl Acad Sci U S A 112:11977-11982.

Yu S, Zhang XE, Chen G, and Liu W. 2012. Compromised cellular responses to DNA damage accelerate chronological aging by incurring cell wall fragility in Saccharomyces cerevisiae. Mol Biol Rep 39:3573-3583. 


\section{Figure 1 (on next page)}

Experimental scheme to measure the exogenous hydrogen peroxide effect on genome instability at the MET15 locus.

Cells were treated with various doses of exogenous hydrogen peroxide, and then transferred to plates of lead-containing media. 


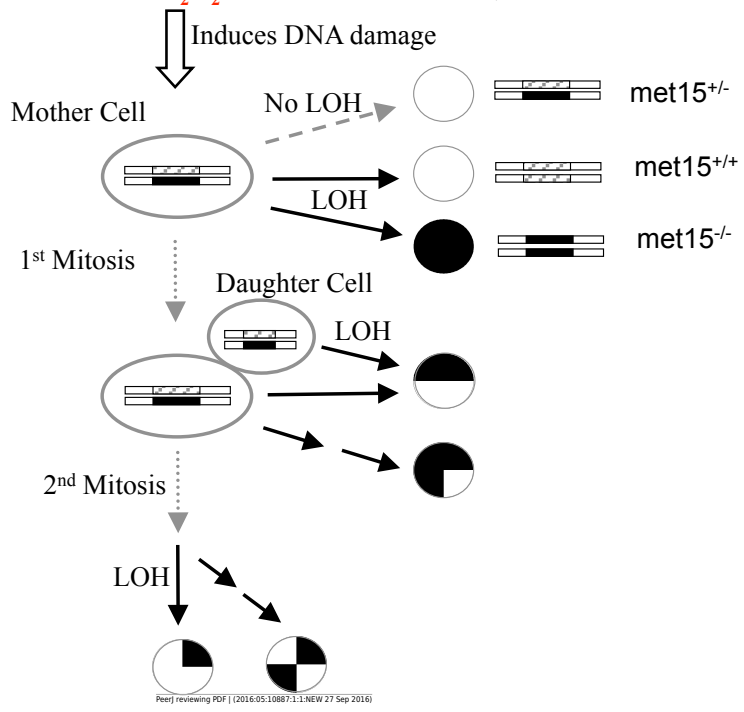




\section{Figure 2 (on next page)}

Exogenous hydrogen peroxide induces dose-dependent increase of loss of heterozygosity and drop of viability.

Experimental results of two strains are presented, with strain YPS163 in (A) and strain $101 \mathrm{~S}$ in (B). The vertical axes indicate the fractions of viabilities, black colonies, and half-black colonies. The concentrations of hydrogen peroxide in the horizontal axes are indicated in percentages estimated by volumes. Dashed bars indicate standard deviations. Red squares indicate the mid-transition points. 
(A)

PeerJ

YPS163 2011 April 13

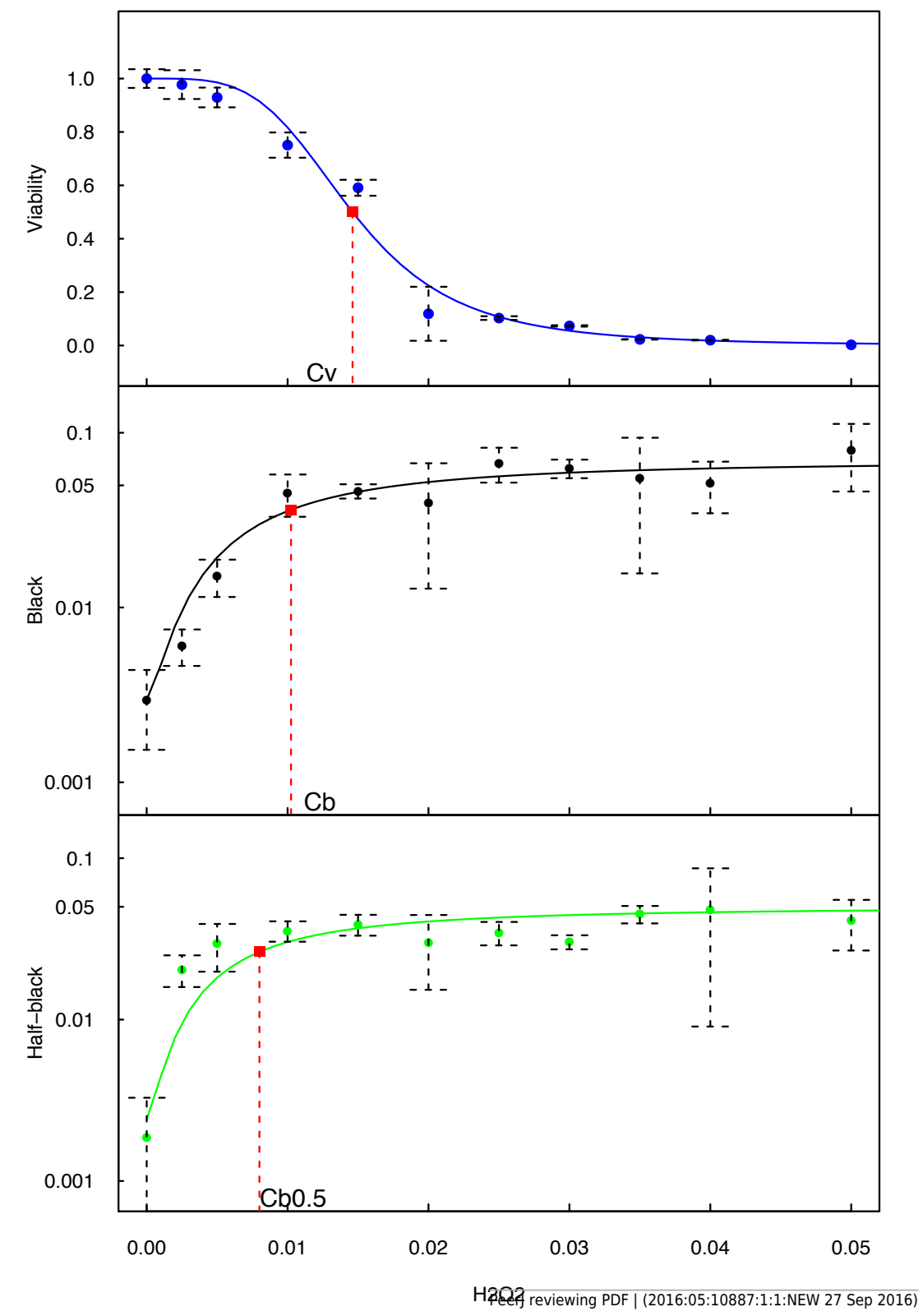

(B) Manuscript to be reviewed

101S 2011 Jan 25

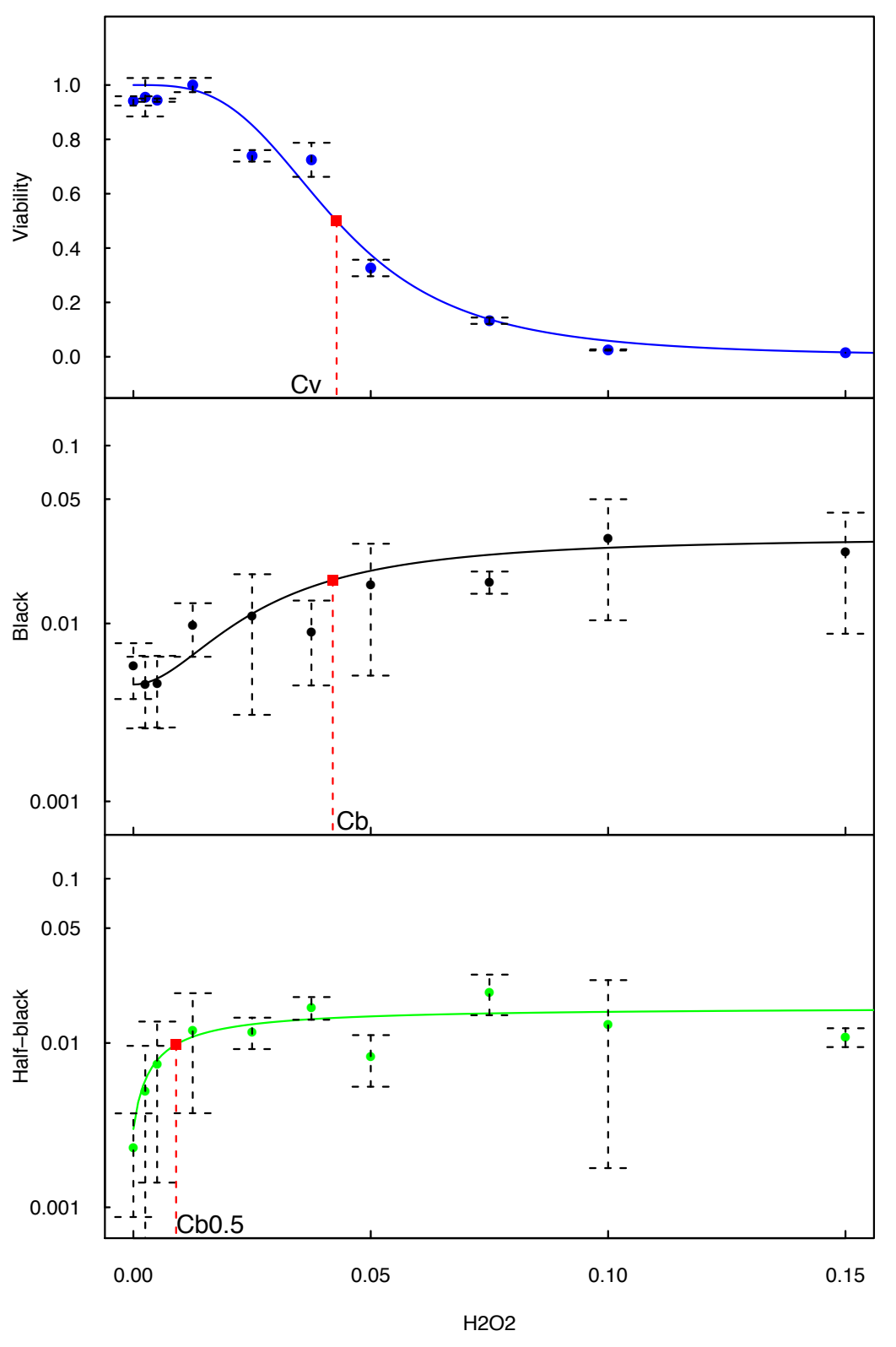


Figure 3 (on next page)

Hydrogen peroxide induced LOH correlates with replicative lifespan and mitotic asymmetry.

(A) Genomic tolerance to hydrogen peroxide induced $\mathrm{LOH}$, as measured by $\mathrm{C}_{\mathrm{b}} / \mathrm{C}_{\mathrm{v}}$, correlates with average replicative lifespan (ARLS). (B) Trade-off between tolerance to hydrogen peroxide induced LOH and mitotic asymmetry. In both panels, blue dots represent the average measurements of yeast strains. The red dashed lines are regression lines. 


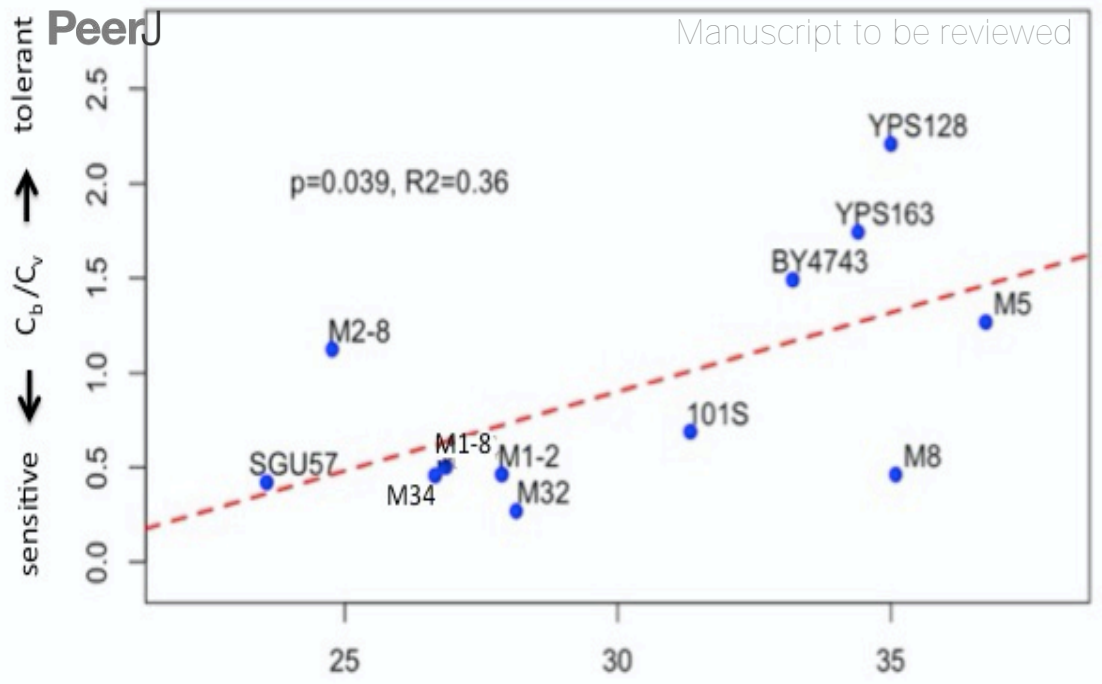

ARLS

(B)

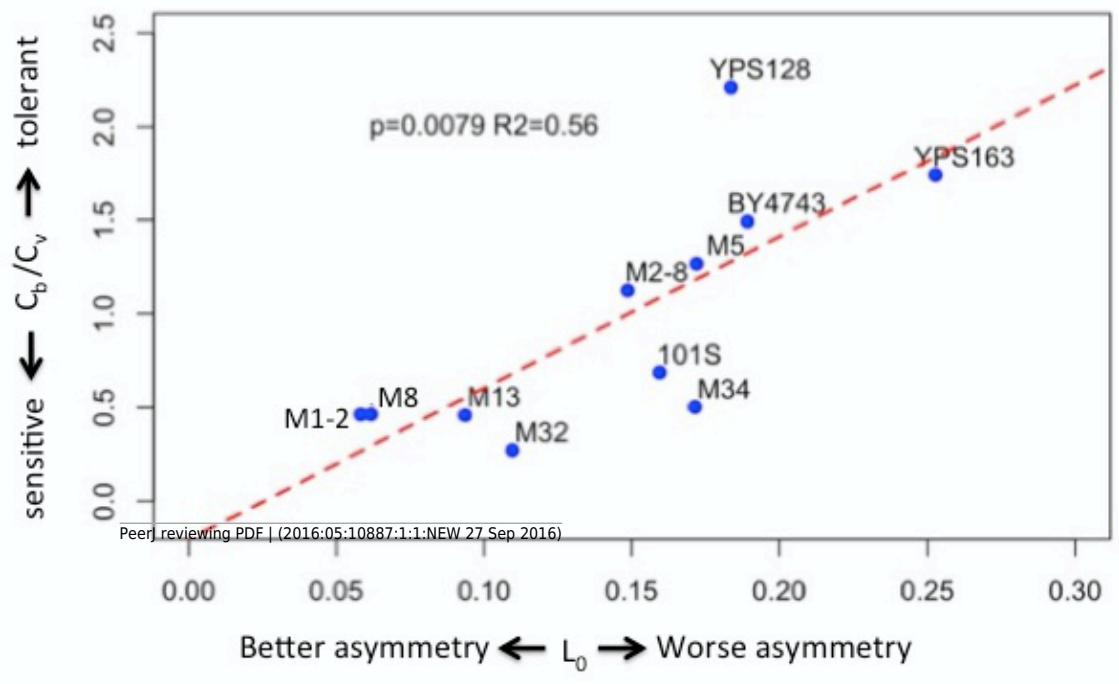




\section{Figure 4 (on next page)}

Contrasting onsets of $\mathrm{LOH}$ events between exogenous hydrogen peroxide treatment and chronological aging and its implications.

(A) The mid-transition points of $\mathrm{LOH}$ frequencies in exogenous hydrogen peroxide treatment and chronological aging. To illustrate the contrast, we used the same viability curve for both exogenous hydrogen peroxide treatment and chronological aging. (B) Bar plots of $C_{b} / C_{v}$ and $T_{g} / T_{c}$ ratios. (C) Scatter plots of $T_{g} / T_{c}$ and $C_{b} / C_{v}$ ratios. (D) Strong negative correlation between mitotic asymmetry $L_{0}$ and $\left(T_{g} / T_{c}\right) /\left(C_{b} / C_{v}\right)$. A) The mid-transition points of $L O H$ frequencies in exogenous hydrogen peroxide treatment and chronological aging. To illustrate the contrast, we used the same viability curve for both exogenous hydrogen peroxide treatment and chronological aging. (B) Bar plots of $\mathrm{Cb} / \mathrm{Cv}$ and $\mathrm{Tg} / \mathrm{Tc}$ ratios. (C) Scatter plots of $\mathrm{Tg} / \mathrm{Tc}$ and $\mathrm{Cb} / \mathrm{Cv}$ ratios. (D) Strong negative correlation between mitotic asymmetry $\mathrm{L}_{0}$ and ( $\mathrm{Tg} / \mathrm{Tc}) /(\mathrm{Cb} / \mathrm{Cv})$. 


\section{Figure 5 (on next page)}

Main conclusions and a plausible model.

(A) Ability to counter genotoxic stress correlates positively with replicative lifespan, but trades-off for mitotic asymmetry. Consequently, the connection between mitotic asymmetry and replicative lifespan is more complicated. (B and C) A plausible model to account for the trade-off between mitotic asymmetry and tolerance to oxidative stress-induced genomic instability. Red stars represent ROS-induced damages. Mother cells tend to prevent ROSinduced damages to themselves, but this sacrifice put their own chromosomal DNA at an increased risk of ROS induced damages. A cell with better mitotic asymmetry is presented in (B) and a cell with poor mitotic asymmetry in (C). Daughter cells have fewer damages to start with in (B) but more damages in (C). Mother cells are at a high risk for LOH in (B) but low risk for $\mathrm{LOH}$ in $(\mathrm{C})$. 
PeerJ

$$
\text { Correlation } \sqrt{\begin{array}{c}
\text { Replicative } \\
\text { Lifespan }
\end{array}}
$$

(B)

A yeast strain with good mitotic asymmetry tends to be sensitive to genotoxic stress.

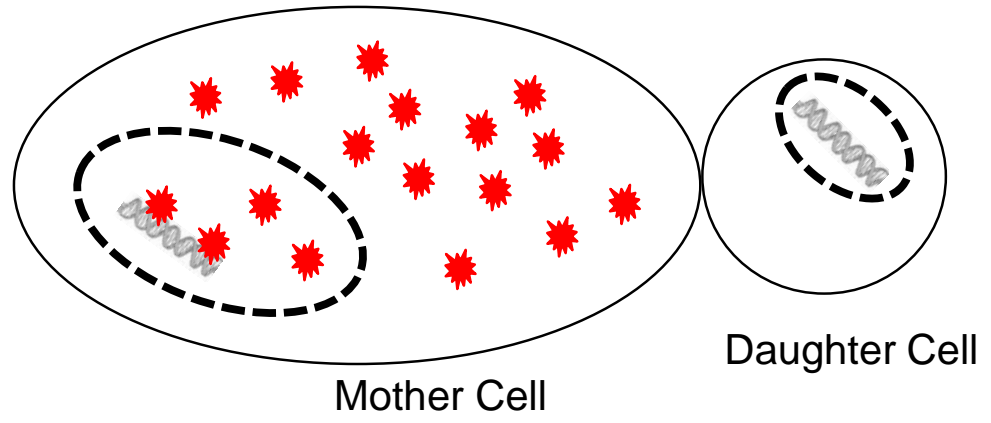

(C)

A yeast strain with poor mitotic asymmetry tends to be more tolerant to genotoxic stress.

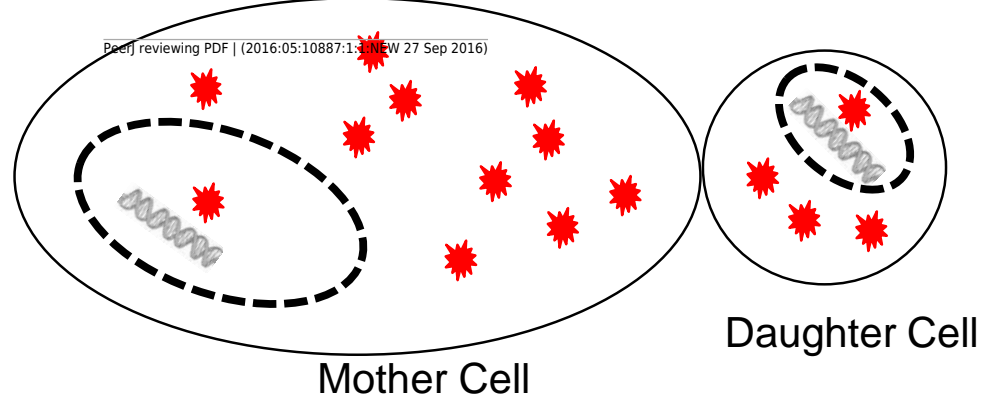

